\title{
Professor Dr. Walter Marget - 60 Jahre
}

Walter Marget wird am 1. August 198060 Jahre alt. Fast die Hälfte seines Lebens sind wir beide miteinander den pädiatrisch-akademischen Weg gegangen. Er kam 1951, ein Jahr nach mir, zu Walter Keller an die Freiburger Universitätskinderklinik; er ging mit mir 1961 von Freiburg nach Tübingen und von dort 1967 nach München. Mit Freude schreibe ich daher dieses Geleitwort zu einem ihm gewidmeten Heft der ,Infection“, wobei es mir ein besonderes Vergnügen bereitet, daß er, der große Editorin-chief der Zeitschrift, nichts von der Drucklegung dieses Heftes weiß.

Walter Keller hatte Walter Marget, der rund 4 Jahre lang bei Prof. Rodenwaldt und bei Prof. Habs in Heidelberg mikrobiologisch ausgebildet worden war, an die Freiburger Kinderklinik geholt, damit er dort eine Mikrobiologie am Krankenbett aufzog, parallel zu der Oskar Vivell anvertrauten Virologie am Krankenbett, - etwas in der damaligen deutschen Pädiatrie ganz Ungewöhnliches. Diese Aufgabe aber wurde Margets Berufung: Er wurde der Promotor der Erforschung und Bekämpfung des infektiösen Hospitalismus in der zeitgenössischen deutschen Pädiatrie und er entwickelte sich zum international herausragenden Fachmann einer wirksamen und rationellen Antibiotika-Anwendung in Klinik und Praxis.

Die heute allerorts so ernst genommenen Hospital-Infektionen begann er schon Ende der 50er Jahre zu bearbeiten - die Coli-Enteritiden der Säuglinge und die Staphylokokken-Hospitalinfektionen (seine Habilitationsschrift) und er hat entscheidende Beiträge zur Epidemiologie und Bekämpfung der ,,modernen“, in der Intensivmedizin auftretenden nosokomialen Infektionen geliefert, mit der an sich verblüffend simplen, aber offensichtlich nicht ohne weiteres klaren Erkenntnis, daß man es weniger mit „,Problemkeimen“ als mit Problempatienten und problematischen technischen Hilfsmitteln zu tun hat. Durch seine Initiative kam eine große Pilotstudie zur Erfassung und systematischen Bekämpfung der Hospitalinfektionen an fünf Kliniken Süddeutschlands zustande. Die Berufung seines Mitarbeiters Franz Daschner 1976 als Klinikhygieniker nach Freiburg war eine schöne - ich möchte sagen: folgerichtige - Bestätigung seines erfolgreichen Wirkens auf diesem Gebiet.

Wegweisend waren auch seine Untersuchungen der Harnwegsinfektionen im Kindesalter, in den 60er Jahren in Tübingen beginnend und in München zu einer großen Studie an über 2000 Säuglingen als Screening und teilweise auch als prospektive Untersuchung ausgebaut. Sie brachte nicht nur eine Fülle von praktischen Ergebnissen hinsichtlich Diagnostik und Therapie, sondern wurde auch Anstoß zu einer Grundlagenforschung über die pathogenetische Bedeutung des Lipoid A des Coliendotoxins für die Pyelonephritis.

Sozusagen Alltagsarbeit war und ist die ständige Prüfung neuer Antibiotika auf Wirksamkeit, Dosierung und Blutspiegel bei Kindern in den verschiedenen Altersstufen, insbesondere bei Neugeborenen und jungen Säuglingen, und die Ermittlung günstiger Antibiotika-Kombinationen, dies alles basierend auf sorgfältigen pharmakokinetischen Studien. Mehrere von Walter Marget in den letzten Jahren auf diesem Arbeitsgebiet durchgeführte internationale Symposien in Sils-Maria haben den hohen Rang dieser Arbeiten deutlich gemacht.

Bis heute ist trotz aller speziellen Wissenschaft aber das Prinzip erhalten geblieben, unter dem Walter Marget einst bei Walter Keller antrat: er wurde, ohne Abstriche an Ausbildung und Dienst, Kinderarzt und er blieb Kinderarzt, als klinischer Oberarzt und als Abteilungsleiter. Die wissenschaftliche Arbeit ist ihm dabei Lebenselixier, das jedem Forscher so wohl bekannte hin und her zwischen Fehlschlägen und Triumphen ein Stimulans zur Arbeit. Walter Marget brachte und bringt dabei das Kunststück fertig, Außerordentliches zu leisten, ohne daß man den Eindruck hat, daß er sich anstrengen muß. Dies liegt an seinem Gespür für das, was wichtig ist, wodurch er kaum je Arbeit und Zeit an Dinge verschwendet, bei denen nichts herauskommt.

Viele Doktoranden haben bei ihm gelernt, Probleme der klinischen Infektiologie und der präventiven und kurativen Infektionsbekämpfung zu sehen und zu bearbeiten; zwei seiner Schüler und Mitarbeiter, Franz Daschner und Dieter Adam, wirken bereits erfolgreich als akademische Lehrer. Die von ihm geleitete Abteilung für Antimikrobielle Therapie und Infektionsimmunologie an unserer Klinik ist Ziel vieler Besucher und von Gastärzten aus dem In- und Ausland. Der planmäßige Stab, der ihm von der Klinik zur Verfügung gestellt werden kann, ist gering, und so erfolgt ein großer Teil der Arbeit mit Drittmitteln. Wir Kliniker - die Pädiater und die Kinderchirurgen des Hauses - können uns eine Arbeit ohne die jederzeit greifbare Hilfe und den Rat dieser Abteilung gar nicht mehr vorstellen. Es ist eine im wahrsten Sinne des Wortes integrierte Abteilung, wissenschaftlich profiliert und eigenständig und dennoch im ganzen Hause präsent und wirksam. Ich bin Walter Marget sehr dankbar, daß er dieses mir als Ideal vorschwebende Prinzip einer Abteilung an einer Klinik so vorbildlich realisiert hat.

Meine Hochachtung dem Arzt und Forscher Walter Marget, meine herzlichen Geburtstagswünsche dem Freunde.

Klaus Betke 